\title{
Controlling inclusive cross sections in parton shower + matrix element merging
}

\section{Simon Plätzer}

DESY,

Notkestrasse 85, D-22607 Hamburg, Germany

E-mail: simon.plaetzer@desy.de

ABSTRACT: We propose an extension of matrix element plus parton shower merging at tree level to preserve inclusive cross sections obtained from the merged and showered sample. Implementing this constraint generates approximate next-to-leading order (NLO) contributions similar to the LoopSim approach. We then show how full NLO, or in principle even higher order, corrections can be added consistently, including constraints on inclusive cross sections to account for yet missing parton shower accuracy at higher logarithmic order. We also show how NLO accuracy below the merging scale can be obtained.

Keywords: Monte Carlo Simulations, NLO Computations

ARXIV EPRINT: 1211.5467 


\section{Contents}

1 Introduction $\quad 1$

$\begin{array}{llr}2 & \text { Setting the scene } & 2\end{array}$

3 Tree-level merging $\quad 4$

$\begin{array}{lll}4 & \text { Inclusive cross sections } & 7\end{array}$

5 Injecting NLO corrections $\quad 9$

6 Corrections below the merging scale $\quad 11$

7 Conclusions and outlook $\quad 12$

\section{Introduction}

Parton shower event generators, [1-3], to name only few of the most recent general-purpose simulations, are by now indispensable workhorses of experimental as well as theoretical studies for comparing (standard model) theoretical predictions to measured observables in a most detailed way. Within recent years, tremendous progress has been made in improving the approximations underlying those simulations by exact calculations in perturbation theory. Starting from simple matrix element corrections on the one hand, improving the hardest parton shower emission to be driven by the exact tree level matrix element $[4,5]$, subsequently tree level matrix elements for $n>1$ hardest emissions have been included consistently [6-13]. These approaches of merging multileg matrix elements at tree level, i.e. leading order (LO), have been accompanied by efforts of matching parton shower simulations and perturbative calculations at next-to-leading order (NLO), including both matrix elements for additional hard emission at tree level as well as virtual, one-loop corrections to the hard process of interest, [14-19]. On the other hand, parton shower algorithms themselves have evolved from crude approximations of multiple parton emission to more and more refined and precise tools [20-26], eventually aiding attempts of combining fixed-order calculations with shower resummation in a most consistent way.

NLO matching has been used to improve tree level merging algorithms, [27-30], but only recently, taking full advantage of developments in both merging, matching and shower algorithms, first experience has been gained in combining NLO QCD corrections to multijet final states of different multiplicity with subsequent parton shower emissions [31-34]. The aim of such approaches is to obtain an event sample which will provide LO (NLO) precision for observables controlled by hard $n$-parton emission as long as exact QCD corrections are included for up to $n$ partons at tree level ( $n$ partons at one loop and $n+1$ partons at tree 
level), while including resummation by means of shower simulation at whatever accuracy is provided by these algorithms.

While merging approaches at tree level have been proven to be amazingly powerful in describing experimental data, and can now be considered to be well understood at a theoretical level (particularly with respect to logarithmic dependence on the merging scale separating hard, matrix element driven emissions from softer parton shower emissions), their generalization to include NLO corrections yet suffers from impact of the merging scale at a level of logarithmic accuracy which by no means can be provided by existing shower algorithms. The most striking signs of this dependence are expected in inclusive cross sections as predicted by such simulations, and proposals to cure the problem by explicit input of resummation results at higher accuracy have been made [35]. In the present contribution we try to approach this problem from a rather pragmatic point of view by setting up a formalism to systematically include higher oder corrections in parton shower simulations, while satisfying constraints to obtain the proper inclusive cross sections at the respective order of perturbative calculations available to the merging algorithm.

\section{Setting the scene}

We will consider a generic parton shower with an evolution variable $q$ (all other kinematic variables of a splitting are suppressed for the sake of readability), which is driven by splitting kernels $P\left(\phi_{n}, q\right)$. Here and in the following, $\phi_{n}$ denotes a partonic configuration (phase space point) containing $n$ additional partons with respect to the lowest order process of interest, and $P\left(\phi_{n}, q\right)$ determines the dynamics of emission at a scale $q$ off the partonic system $\phi_{n}$. The Sudakov form factor associated to $P\left(\phi_{n}, q\right)$, evolving from a hard scale $Q$ to a soft scale $q$, is given by

$$
\Delta_{n}(q \mid Q)=\exp \left(-\int_{q}^{Q} \mathrm{~d} k \frac{\mathrm{d} \phi_{n+1}}{\mathrm{~d} \phi_{n} \mathrm{~d} k} P\left(\phi_{n}, k\right)\right),
$$

with the phase space Jacobian $\mathrm{d} \phi_{n+1} / \mathrm{d} \phi_{n}$ depending implicitly on the shower kinematic variables. Partonic configurations $\phi_{n}$ are determined according to differential cross sections $\mathrm{d} \sigma\left(\phi_{n}, q_{n}|\cdots| q_{0}\right)$, where the sequence of scales $q_{n}<q_{n-1}, \ldots, q_{0}$ is either directly determined from shower evolution, or is assigned by a clustering procedure corresponding to the inverse of a possible shower evolution $\phi_{0} \rightarrow \phi_{n}$, if the cross section is determined by exact matrix elements. ${ }^{1}$ In the case of exact matrix elements, reweighting to account for couplings and PDF factors evaluated at the clustering scales may as well be included in this notation. For the sake of readability we will skip the whole sequence of scales and denote $\mathrm{d} \sigma\left(\phi_{n}, q_{n}|\ldots| q_{0}\right)=\mathrm{d} \sigma\left(\phi_{n}, q_{n}\right)$. The parton shower action on cross sections for $\phi_{n}$

\footnotetext{
${ }^{1}$ Note that ordered histories may not always exist. In these cases we can assume that, for example, the history with the longest ordered history from smaller to larger scales has been chosen. This will not pose a problem for the formalism outlined here, provided we set Sudakov form factors with unordered scales equal to one, $\Delta_{n}(Q \mid q)=1$ for $Q>q$. Also note that a clustering down to the lowest order process may not always be possible, in case of which we terminate the clustering sequence at the last possible clustering step.
} 
events is given by ${ }^{2}$

$$
\begin{aligned}
\operatorname{PS}_{\mu}\left[\mathrm{d} \sigma\left(\phi_{n}, q_{n}\right)\right]=\mathrm{d} \sigma( & \left.\phi_{n}, q_{n}\right) \Delta_{n}\left(\mu \mid q_{n}\right) \\
& +\mathrm{PS}_{\mu}\left[\mathrm{d} \sigma\left(\phi_{n}, q_{n}\right) \frac{\mathrm{d} \phi_{n+1}}{\mathrm{~d} \phi_{n}} P_{\mu}\left(\phi_{n}, q_{n+1}\right) \Delta_{n}\left(q_{n+1} \mid q_{n}\right)\right] .
\end{aligned}
$$

The first contribution are events where no further radiation has been generated ( $\mu$ denotes the parton shower cutoff and the splitting kernels $P_{\mu}\left(\phi_{n}, q_{n+1}\right)=P\left(\phi_{n}, q_{n+1}\right) \theta\left(q_{n+1}-\mu\right)$ vanish for scales below the cutoff), while the second contribution corresponds to at least one emission.

Considering parton showering off the lowest order tree level cross section, $\mathrm{d} \sigma^{(0)}\left(\phi_{0}, q_{0}\right)$, we find that the first $N$ iterations of the parton shower action give rise to

$$
\begin{aligned}
& \operatorname{PS}_{\mu}\left[\mathrm{d} \sigma^{(0)}\left(\phi_{0}, q_{0}\right)\right] \\
& =\mathrm{d} \sigma^{(0)}\left(\phi_{0}, q_{0}\right) \sum_{k=0}^{N-1} \frac{\mathrm{d} \phi_{k}}{\mathrm{~d} \phi_{0}}\left(\prod_{i=1}^{k} P_{\mu}\left(\phi_{i-1}, q_{i}\right)\right) \Delta_{k}\left(\mu\left|q_{k}\right| \cdots \mid q_{0}\right) \\
& \quad+\mathrm{PS}_{\mu}\left[\mathrm{d} \sigma^{(0)}\left(\phi_{0}, q_{0}\right) \frac{\mathrm{d} \phi_{N}}{\mathrm{~d} \phi_{0}} P_{\mu}\left(\phi_{N-1}, q_{N}\right) \cdots P_{\mu}\left(\phi_{0}, q_{1}\right) \Delta_{N-1}\left(q_{N}|\cdots| q_{0}\right)\right]
\end{aligned}
$$

where $\Delta_{k}\left(\mu\left|q_{k}\right| \cdots \mid q_{0}\right)=\Delta_{k}\left(\mu \mid q_{k}\right) \cdots \Delta_{0}\left(q_{1} \mid q_{0}\right)$. Considering the exclusive and inclusive cross sections for producing exactly $n$ or at least $n$ partons, respectively, we have

$$
\begin{array}{ll}
=n & \mathrm{~d} \sigma^{(0)}\left(\phi_{0}, q_{0}\right) \frac{\mathrm{d} \phi_{n}}{\mathrm{~d} \phi_{0}} P_{\mu}\left(\phi_{n-1}, q_{n}\right) \cdots P_{\mu}\left(\phi_{0}, q_{1}\right) \Delta_{n}\left(\mu\left|q_{n}\right| \cdots \mid q_{0}\right) \\
\geq n & \mathrm{~d} \sigma^{(0)}\left(\phi_{0}, q_{0}\right) \frac{\mathrm{d} \phi_{n}}{\mathrm{~d} \phi_{0}} P_{\mu}\left(\phi_{n-1}, q_{n}\right) \cdots P_{\mu}\left(\phi_{0}, q_{1}\right) \Delta_{n-1}\left(q_{n}|\cdots| q_{0}\right) .
\end{array}
$$

Note that the latter expression is a direct implication of the parton shower being unitary. In particular, the total inclusive cross section is not altered by the parton shower and determined by the lowest order 'input' cross section $\mathrm{d} \sigma^{(0)}$, as would be expected from the very definition of an inclusive cross section.

This property is a direct result of the fact that the emission contribution of the parton shower at scale $q$ is an exact differential of the no emission probability down to $q$,

$$
\int_{q}^{q_{k-1}} \mathrm{~d} q_{k} \frac{\mathrm{d} \phi_{k}}{\mathrm{~d} \phi_{k-1} \mathrm{~d} q_{k}} P\left(\phi_{k-1}, q_{k}\right) \Delta_{k-1}\left(q_{k} \mid q_{k-1}\right)=1-\Delta_{k-1}\left(q \mid q_{k-1}\right) .
$$

Introducing $P_{\mu \mid \rho}\left(\phi_{k-1}, q_{k}\right)=P\left(\phi_{k-1}, q_{k}\right) \theta\left(\rho-q_{k}\right) \theta\left(q_{k}-\mu\right)$ with $\rho>\mu$, and associating $\mathrm{PS}_{\mu \mid \rho}$ as the associated action, we also have

$$
\mathrm{PS}_{\mu}[\cdot]=\operatorname{PS}_{\mu \mid \rho}\left[\mathrm{PS}_{\rho}[\cdot]\right]
$$

i.e. we can always split the shower evolution into two (or more) scale ranges. We illustrate the main formulae discussed in this section in a diagramatic manner in figure 1 .

\footnotetext{
${ }^{2}$ For the following note that PS[.] is linear.
} 


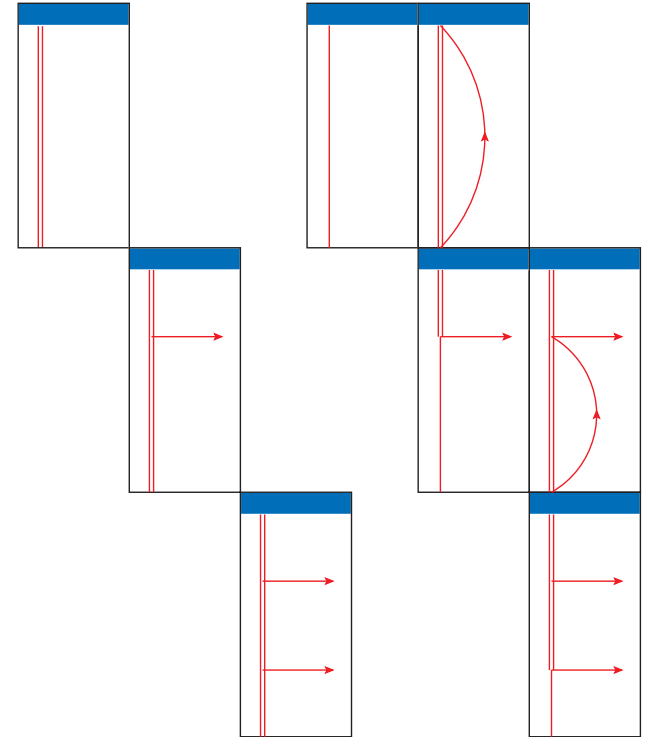

(a)

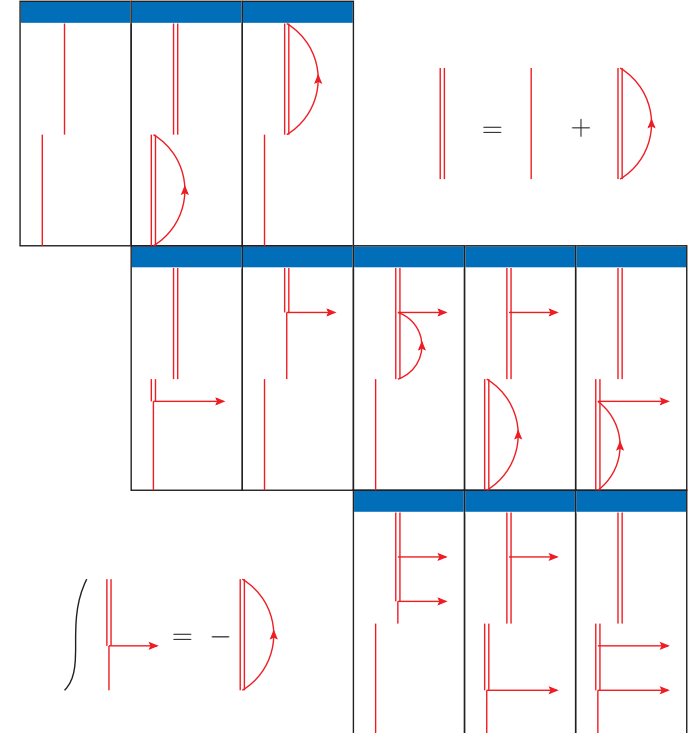

(c)

Figure 1. Main properties of parton showers illustrated in a diagrammatic way. Each box with a black frame denotes a $n$-parton contribution, where $n$ runs from top to bottom. Rows need to be summed to obtain the total cross section driving a given parton multiplicity. The parton shower evolves along the vertical red lines from larger (top) to smaller (bottom) scales, with each single line representing an evolution scale interval available to emission of additional partons, with an associated factor of one. Double red lines denote the Sudakov factors associated to a given evolution interval, horizontal arrows branching off denote emission at a certain scale and indicate multiplication with the respective splitting kernel. Integrations over the product of a splitting kernel and Sudakov form factor, as well as the graphical representation of eq. (2.4) are defined in the lower left and upper right corners of part (c), respectively. With the parton shower cutoff at the bottom of each box, part (a) shows exclusive cross sections for zero, one and two partons emitted, cf. eq. (2.3). Part (b) illustrates the shower cross section integrated over the contributions of more than two emissions, making use of eq. (2.4); the form of inclusive cross sections is obvious, as each column of two boxes integrates to zero. Part (c) illustrates how the shower splits up into two evolution intervals, cf. eq. (2.5).

\section{Tree-level merging}

Having analyzed the exclusive and inclusive cross sections for $n$-parton production by the shower, we will now review how these predictions can be improved by including higher multiplicity tree-level matrix elements. Clearly, the desired result in terms of exclusive $n$ parton states is to replace the product of splitting kernels approximating an exact tree-level matrix element by the full answer. To this extent, in the presence of a maximum number of $N$ additional partons to be described by matrix elements, we are therefore to satisfy a merging condition

$$
\begin{aligned}
\operatorname{PS}_{\mu}\left[\mathrm{d} \sigma_{N, \mu}^{\text {merged }}\right]= & \sum_{k=0}^{N-1} \mathrm{~d} \sigma_{\mu}^{(0)}\left(\phi_{k}, q_{k}\right) \Delta_{k}\left(\mu\left|q_{k}\right| \cdots \mid q_{0}\right) \\
& \quad+\operatorname{PS}_{\mu}\left[\mathrm{d} \sigma_{\mu}^{(0)}\left(\phi_{N}, q_{N}\right) \Delta_{N-1}\left(q_{N}|\cdots| q_{0}\right)\right] .
\end{aligned}
$$


As is the case for plain parton shower emissions, there are no emissions with a scale below the shower cutoff $\mu$, which we here apply to regulate the divergences in the additional tree-level matrix elements considered. For this reason we have introduced $\mathrm{d} \sigma_{\mu}^{(0)}\left(\phi_{n}, q_{n}\right)=$ $\mathrm{d} \sigma^{(0)}\left(\phi_{n}, q_{n}\right) \theta\left(q_{n}-\mu\right)$.

It is straightforward to show that the merging condition can be satisfied by

$$
\begin{aligned}
\mathrm{d} \sigma_{N, \mu}^{\text {merged }}= & \mathrm{d} \sigma\left(\phi_{0}, q_{0}\right) \\
& +\sum_{k=1}^{N}\left(\mathrm{~d} \sigma_{\mu}^{(0)}\left(\phi_{k}, q_{k}\right)-\frac{\mathrm{d} \phi_{k}}{\mathrm{~d} \phi_{k-1}} P_{\mu}\left(\phi_{k-1}, q_{k}\right) \mathrm{d} \sigma_{\mu}^{(0)}\left(\phi_{k-1}, q_{k-1}\right)\right) \Delta_{k-1}\left(q_{k}|\cdots| q_{0}\right) .
\end{aligned}
$$

Note that we are free to introduce a merging scale $\rho>\mu$ by acting the parton shower on $\mathrm{d} \sigma_{N, \rho}^{\text {merged }}$. The result is similar to eq. (3.1), upon replacing

$$
\begin{array}{r}
\mathrm{d} \sigma_{\mu}^{(0)}\left(\phi_{k}, q_{k}\right) \rightarrow \quad \mathrm{d} \sigma_{\rho}^{(0)}\left(\phi_{k}, q_{k}\right)+\sum_{l=1}^{k} P_{\mu \mid \rho}\left(\phi_{k-1}, q_{k}\right) \cdots P_{\mu \mid \rho}\left(\phi_{k-l}, q_{k-l+1}\right) \times \\
\times \frac{\mathrm{d} \phi_{k}}{\mathrm{~d} \phi_{k-l}} \mathrm{~d} \sigma_{\rho}^{(0)}\left(\phi_{k-l}, q_{k-l}\right)
\end{array}
$$

i.e. emissions at scales smaller than $\rho$ are purely driven by shower dynamics, whereas above the exact tree level matrix element is used. The merging scale at this point can be viewed as just an efficiency tweak for not having to evaluate exact matrix elements in a region where they are well approximated by the shower.

For later purposes, and to make the connection to CKKW-type merging [6-13] explicit, we rewrite the shower cross section in presence of the merging scale $\rho$ as

$$
\begin{aligned}
\operatorname{PS}_{\mu}\left[\mathrm{d} \sigma_{N, \rho}^{\text {merged }}\right]=\sum_{k=0}^{N-1} \operatorname{PS}_{\mu \mid \rho} & {\left[\mathrm{d} \sigma_{\rho}^{(0)}\left(\phi_{k}, q_{k}\right) \Delta_{k}\left(\rho\left|q_{k}\right| \cdots \mid q_{0}\right)\right] } \\
+ & \operatorname{PS}_{\mu}\left[\mathrm{d} \sigma_{\rho}^{(0)}\left(\phi_{N}, q_{N}\right) \Delta_{N-1}\left(q_{N}|\cdots| q_{0}\right)\right] .
\end{aligned}
$$

This result follows directly from the merging condition by performing the replacement given in eq. (3.3), and factoring the product of Sudakov form factors at the merging scale $\rho$. All but the highest multiplicity matrix elements are reweighted by Sudakov form factors to account for the fact that there was no emission above the merging scale $\rho$, and showering off these configurations is restricted to emissions below the merging scale. The highest multiplicity available, $N$, receives Sudakov reweighting down the last emission scale, and showering off events with the maximum number of matrix element driven partons is not restricted. Using eq. (2.5), the previous result is simply given by

$$
\begin{aligned}
\operatorname{PS}_{\mu}\left[\mathrm{d} \sigma_{N, \rho}^{\text {merged }}\right]=\operatorname{PS}_{\mu \mid \rho}\left[\sum_{k=0}^{N-1} \mathrm{~d} \sigma_{\rho}^{(0)}(\right. & \left.\phi_{k}, q_{k}\right) \Delta_{k}\left(\rho\left|q_{k}\right| \cdots \mid q_{0}\right) \\
& \left.+\operatorname{PS}_{\rho}\left[\mathrm{d} \sigma_{\rho}^{(0)}\left(\phi_{N}, q_{N}\right) \Delta_{N-1}\left(q_{N}|\cdots| q_{0}\right)\right]\right] .
\end{aligned}
$$


In the merged sample, cross sections for exclusive $n \leq N$ parton configurations above the merging scale $\rho$ are determined by the respective tree level matrix elements including the proper Sudakov suppression, while exclusive cross sections for $n$ partons down to the shower cutoff are determined by a mixture of matrix elements and shower splitting functions depending on which part of the relevant scale sequence is below or above the merging scale. The proper Sudakov suppression is as well retained in the latter case.

Note that the merged cross section and its showered counterpart, eq. (3.5), cover several approaches to tree level merging so long as the merging resolution coincides with the parton shower resolution in case of which no issues with truncated/vetoed showers $[11,12]$ do appear. Throughout the paper we will assume that this is always the case; a mismatch between merging and shower resolution does not pose a conceptual problem to the algorithm proposed in the following. In the presence of a merging scale $\rho$, the formalism outlined here covers the standard merging algorithms of reweighted tree level matrix elements with showering below the merging scale. In this case, upon putting $P_{\rho} \rightarrow 0$ except for emissions off the highest multiplicity, the shower subtractions are absent in $\mathrm{d} \sigma^{\operatorname{merged}_{N, \rho}}$. If no merging scale is present, iterated matrix element corrections are contained in the master formula upon replacing the shower splitting kernels for emissions off a system of up to $N-1$ partons by the respective ratio of tree level matrix elements. In this case, the merged cross section coincides with the lowest order cross section. To work in a most generic setup, we will stay with the general solution to the merging condition, which does not require any modification to the shower acting downstream the hard processes obtained from the merged cross section.

For convenience, let us introduce the functional

$$
\operatorname{PS}_{\mu}^{-1}\left[\mathrm{~d} \sigma_{\mu}\left(\phi_{n}, q_{n}\right)\right]=\frac{\mathrm{d} \sigma_{\mu}\left(\phi_{n}, q_{n}\right)}{\Delta_{n}\left(\mu \mid q_{n}\right)}-\frac{\mathrm{d} \phi_{n+1}}{\mathrm{~d} \phi_{n}} P_{\mu}\left(\phi_{n}, q_{n+1}\right) \frac{\mathrm{d} \sigma_{\mu}\left(\phi_{n}, q_{n}\right)}{\Delta_{n}\left(\mu \mid q_{n+1}\right)}
$$

which satisfies

$$
\begin{aligned}
\mathrm{PS}_{\mu}\left[\mathrm{PS}_{\mu}^{-1}\left[\mathrm{~d} \sigma_{\mu}\left(\phi_{n}, q_{n}\right)\right]\right] & =\mathrm{d} \sigma_{\mu}\left(\phi_{n}, q_{n}\right) \\
\text { and } \quad \operatorname{PS}_{\mu}\left[\operatorname{PS}_{\rho}^{-1}\left[\mathrm{~d} \sigma_{\rho}\left(\phi_{n}, q_{n}\right)\right]\right] & =\mathrm{PS}_{\mu \mid \rho}\left[\mathrm{d} \sigma_{\rho}\left(\phi_{n}, q_{n}\right)\right]
\end{aligned}
$$

Using this definition, we can rewrite the merged cross section as

$$
\mathrm{d} \sigma_{N, \rho}^{\text {merged }}=\sum_{k=0}^{N-1} \operatorname{PS}_{\rho}^{-1}\left[\mathrm{~d} \sigma_{\rho}^{(0)}\left(\phi_{k}, q_{k}\right) \Delta_{k}\left(\rho\left|q_{k}\right| \cdots \mid q_{0}\right)\right]+\mathrm{d} \sigma_{\rho}^{(0)}\left(\phi_{N}, q_{N}\right) \Delta_{N-1}\left(q_{N}|\cdots| q_{0}\right),
$$

which summarizes the solution to the tree level merging condition in a most transparent way. Being of purely formal use for the present letter, it is worth noting that $\mathrm{PS}_{\rho}^{-1}$ facilitates the subtraction of parton shower contributions above the scale $\rho$ at all orders. Turning this observation around, it can actually be used as a generating functional for subtractions needed to satisfy fixed-order matching conditions upon expanding to the respective order. 
Indeed,

$$
\begin{aligned}
\operatorname{PS}_{\mu}^{-1}\left[\mathrm{~d} \sigma_{\mu}\left(\phi_{n}, q_{n}\right)\right]= & \mathrm{d} \sigma_{\mu}\left(\phi_{n}, q_{n}\right)\left(1+\int_{\mu}^{q_{n}} \mathrm{~d} q_{n+1} \frac{\mathrm{d} \phi_{n+1}}{\mathrm{~d} \phi_{n} \mathrm{~d} q_{n+1}} P_{\mu}\left(\phi_{n}, q_{n+1}\right)\right) \\
& -\frac{\mathrm{d} \phi_{n+1}}{\mathrm{~d} \phi_{n}} P_{\mu}\left(\phi_{n}, q_{n+1}\right) \mathrm{d} \sigma_{\mu}\left(\phi_{n}, q_{n}\right)+\mathcal{O}\left(\alpha_{s}^{2}\right)
\end{aligned}
$$

is readily identified as the corrections required for NLO matching. Note also that the action of $\mathrm{PS}_{\rho}^{-1}$ can actually be implemented in a Monte Carlo simulation by performing the veto algorithm from smaller to larger scales while using a simple prescription to exponentiate $-P\left(\phi_{n}, q\right)$ at the expense of introducing weighted events [36].

\section{Inclusive cross sections}

Let us now turn to inclusive cross sections. We are free to split up the phase space integration into a region of scales below the merging scale $\rho$, and one above. Performing the integration over smaller scales first (or, equivalently, considering jet cross sections in a jet measure exactly corresponding to inverting the parton shower), we effectively remove the shower action $\mathrm{PS}_{\mu \mid \rho}$ from eq. (3.5).

Looking at inclusive cross sections of at least $n \leq N$ jets, we face essentially a nonunitary evolution due to the matrix elements not coinciding with products of splitting kernels anymore. While for the highest multiplicity we find the same pattern as present in the parton shower by the very definition of the merging condition,

$$
\geq N \quad \mathrm{~d} \sigma_{\rho}^{(0)}\left(\phi_{N}, q_{N}\right) \Delta_{N-1}\left(q_{N}|\cdots| q_{0}\right)
$$

the result for one parton less does not anymore resemble the functional dependence present in the shower: ${ }^{3}$

$$
\begin{aligned}
& \geq N-1 \\
& \mathrm{~d} \sigma_{\rho}^{(0)}\left(\phi_{N-1}, q_{N-1}\right) \Delta_{N-2}\left(q_{N-1}|\cdots| q_{0}\right) \\
& \quad+\int_{\rho}^{q_{N-1}} \mathrm{~d} q_{N}\left(\frac{\mathrm{d} \sigma_{\rho}^{(0)}\left(\phi_{N}, q_{N}\right)}{\mathrm{d} q_{N}}-\frac{\mathrm{d} \phi_{N}}{\mathrm{~d} \phi_{N-1} \mathrm{~d} q_{N}} P_{\rho}\left(\phi_{N-1}, q_{N}\right) \mathrm{d} \sigma_{\rho}^{(0)}\left(\phi_{N-1}, q_{N-1}\right)\right) \times \\
& \times \Delta_{N-1}\left(q_{N}|\cdots| q_{0}\right) .
\end{aligned}
$$

In order to restore the form of the cross section obtained by the shower, we need to subtract the second term obtained for the inclusive $N-1$ jet cross section. This pattern then continues, and one possibility to solve the issues with inclusive cross sections is then

\footnotetext{
${ }^{3}$ We have added and subtracted the contribution from a shower emission off the $N-1$ parton state, where the positive term has been absorbed by the integration over the scale $q_{N}$ to yield the Sudakov form factor in the first line.
} 


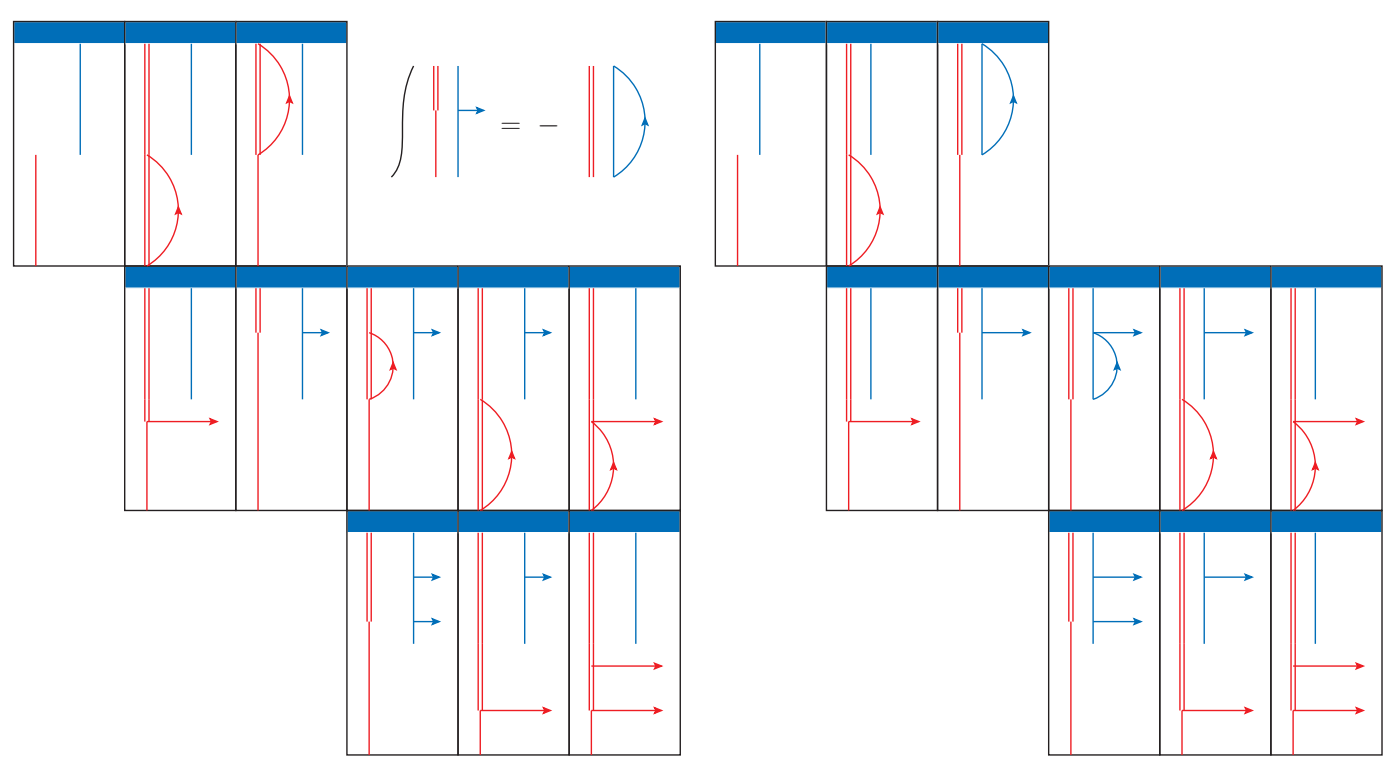

Figure 2. The left panel shows the solution to the merging condition for $N=2$, eq. (3.5), with inconsistencies in inclusive cross sections made explicit. The right panel shows the cross sections obtained from the merged sample upon including counter terms to restore inclusive cross sections as given by eq. (4.2). Here, emissions calculated according to exact tree level matrix elements are made explicit by arrows branching off blue lines, as compared to splitting kernel factors times lower order cross sections associated to arrows branching off red lines. Similar to figure 1, integrations over scale intervals are denoted by half-circles which 'loop' emissions.

given by the replacement

$$
\begin{aligned}
& \mathrm{d} \sigma_{N, \rho}^{\text {merged }} \rightarrow \\
& \mathrm{d} \sigma_{N, \rho}^{\text {merged }}-\sum_{k=0}^{N-1} \mathrm{PS}_{\rho}^{-1}\left[\int _ { \rho } ^ { q _ { k } } \mathrm { d } q _ { k + 1 } \left(\frac{\mathrm{d} \sigma_{\rho}^{(0)}\left(\phi_{k+1}, q_{k+1}\right)}{\mathrm{d} q_{k+1}}\right.\right. \\
& \left.\left.\quad-\frac{\mathrm{d} \phi_{k+1}}{\mathrm{~d} \phi_{k} \mathrm{~d} q_{k+1}} P\left(\phi_{k}, q_{k+1}\right) \mathrm{d} \sigma_{\rho}^{(0)}\left(\phi_{k}, q_{k}\right)\right) \Delta_{k}\left(q_{k+1}|\cdots| q_{0}\right)\right] \\
& =\sum_{k=0}^{N-1} \mathrm{PS}_{\rho}^{-1}\left[\left(\mathrm{~d} \sigma_{\rho}^{(0)}\left(\phi_{k}, q_{k}\right)\right]\right. \\
& \left.\left.\quad-\int_{\rho}^{q_{k}} \mathrm{~d} q_{k+1} \frac{\mathrm{d} \sigma_{\rho}^{(0)}\left(\phi_{k+1}, q_{k+1}\right)}{\mathrm{d} q_{k+1}} \Delta_{k}\left(q_{k+1} \mid q_{k}\right)\right) \Delta_{k-1}\left(q_{k}|\cdots| q_{0}\right)\right] \\
& \quad \mathrm{d} \sigma_{\rho}^{(0)}\left(\phi_{N}, q_{N}\right) \Delta_{N-1}\left(q_{N}|\cdots| q_{0}\right) .
\end{aligned}
$$

The solution to the merging condition as discussed in section 3 , as well as the results obtained from the merged sample including corrections for inclusive cross sections are depicted in figure 2. One remark is in order here: while we have now achieved to retain the expected form of inclusive cross sections (including the constraint that the total inclusive cross section is given by the lowest order input cross section) the exclusive cross sections 
with $n \leq N$ partons above the merging scale take a seemingly different form,

$$
\operatorname{PS}_{\mu \mid \rho}\left[\left(\mathrm{d} \sigma_{\rho}^{(0)}\left(\phi_{n}, q_{n}\right)-\int_{\rho}^{q_{n}} \mathrm{~d} q_{n+1} \frac{\mathrm{d} \sigma_{\rho}^{(0)}\left(\phi_{n+1}, q_{n+1}\right)}{\mathrm{d} q_{n+1}} \Delta_{n}\left(q_{n+1} \mid q_{n}\right)\right) \Delta_{n-1}\left(q_{n}|\cdots| q_{0}\right)\right] .
$$

It is clear that such a change will occur when trying to restore inclusive cross sections. We stress however that the form encountered here does not pose a problem in terms of logarithmic accuracy, as long as the shower kernels are truly capable of reproducing the singly-unresolved limits of the tree level matrix elements. In this case the dominant contributions of integral in the second term will render those singularities to precisely integrate to $\mathrm{d} \sigma_{\rho}^{(0)}\left(\phi_{n}, q_{n}\right)\left(1-\Delta_{n}\left(\rho \mid q_{n}\right)\right)$, restoring the expected Sudakov suppression.

\section{$5 \quad$ Injecting NLO corrections}

Having improved the parton shower by tree level matrix elements, including constraints on inclusive cross sections, we can now turn to the case of including NLO corrections, which we assume are here available up to $M$ additional partons with respect to the lowest order process.

Before discussing this in greater detail, let us consider the respective contributions obtained from the merged sample with corrections for inclusive cross sections. The cross sections to be analyzed are the contributions of exactly $n$ partons above the merging scale (integrated over phase space below $\rho$ ), which we will have to correct to be driven by the respective NLO cross sections. The exclusive cross sections for $n$ partons above the merging scale are given by

$$
\mathrm{d} \sigma_{n, \text { excl }}^{\mathrm{nLO}}=\Delta_{n-1}\left(q_{n}|\cdots| q_{0}\right)\left(\mathrm{d} \sigma_{\rho}^{(0)}\left(\phi_{n}, q_{n}\right)-\int_{\rho}^{q_{n}} \mathrm{~d} q_{n+1} \frac{\mathrm{d} \sigma_{\rho}^{(0)}\left(\phi_{n+1}, q_{n+1}\right)}{\mathrm{d} q_{n+1}} \Delta_{n}\left(q_{n+1} \mid q_{n}\right)\right) .
$$

Note that this does contain the Sudakov form factor for obtaining at least $n$ partons above the merging scale. By the discussion in the previous section, the contribution in brackets will also provide for the remaining Sudakov factor down to the merging scale. Note also, that upon expanding the contribution in brackets to first order in $\alpha_{s}$, we obtain the approximated, exclusive NLO cross section for $n$ jets above a resolution $\rho$ as given by the LoopSim prescription [37], which motivated the label nLO.

This observation directly implies that, if we add a correction cross section given by the inclusive NLO corrections,

$$
\operatorname{PS}_{\rho}^{-1}\left[\left(\mathrm{~d} \sigma_{\rho}^{(1)}\left(\phi_{n}, q_{n}\right)+\int_{0}^{q_{n}} \mathrm{~d} q_{n+1} \frac{\mathrm{d} \sigma^{(0)}\left(\phi_{n+1}, q_{n+1}\right)}{\mathrm{d} q_{n+1}} \theta\left(q_{n}-\rho\right)\right) \Delta_{n-1}\left(q_{n}|\cdots| q_{0}\right)\right],
$$

we will obtain NLO accuracy for exclusive $n$ parton configurations above the merging scale,

$$
\begin{aligned}
\mathrm{d} \sigma_{n, \text { excl }}^{\mathrm{NLO}}=\Delta_{n-1}\left(q_{n}|\cdots| q_{0}\right)\left(\mathrm{d} \sigma_{\rho}^{(0)}(\right. & \left.\phi_{n}, q_{n}\right)+\mathrm{d} \sigma_{\rho}^{(1)}\left(\phi_{n}, q_{n}\right) \\
& \left.+\int_{0}^{\rho} \mathrm{d} q_{n+1} \frac{\mathrm{d} \sigma^{(0)}\left(\phi_{n+1}, q_{n+1}\right)}{\mathrm{d} q_{n+1}} \theta\left(q_{n}-\rho\right)+\mathcal{O}\left(\alpha_{s}^{2}\right)\right) .
\end{aligned}
$$


The Sudakov weight attached to the correction cross section is not running down to the merging scale, as one would naively expect. In this case, a double counting of logarithms of the merging scale would have happened between the nLO cross section and the full one-loop correction. Indeed, the role of the correction we apply here is to precisely replace the first order virtual approximation stemming from expanding the Sudakov form factor by its exact counterpart. Fulfilling this condition is at the heart of the merging efforts at NLO presented so far, [31-34].

Let us stress the importance of maintaining inclusive cross sections in the case of NLO corrections. The problematic contribution by which the inclusive cross section differs from its expected value in the case of tree level merging, as discussed in section. 4 , is given by

$$
\begin{aligned}
\delta\left(\mathrm{d} \sigma_{n, \text { incl }}\right)=\int_{\rho}^{q_{n}} \mathrm{~d} q_{n+1}\left(\frac{\mathrm{d} \sigma_{\rho}^{(0)}\left(\phi_{n+1}, q_{n+1}\right)}{\mathrm{d} q_{n+1}}\right. & \\
& \left.\quad-\frac{\mathrm{d} \phi_{n+1}}{\mathrm{~d} \phi_{n} \mathrm{~d} q_{n+1}} P_{\rho}\left(\phi_{n}, q_{n+1}\right) \mathrm{d} \sigma_{\rho}^{(0)}\left(\phi_{n}, q_{n}\right)\right) \Delta_{n}\left(q_{n+1}|\cdots| q_{0}\right) .
\end{aligned}
$$

This cross section could well be expected not to contribute logarithmically enhanced terms, provided the shower is a good approximation to singly unresolved limits of tree level matrix elements. Upon replacing the LO exclusive cross sections above the merging scale by their NLO counterparts, the above argument does not apply anymore owing to the case that NLO corrections to the shower splitting kernels are not considered. We therefore expect violations of inclusive cross sections at a level of logarithmic approximation which is not covered by the shower anymore. Note that if corrections are only available to the lowest order process $n=0$, then the constraints on merged inclusive cross sections will in this case ensure that also the inclusive NLO cross section is preserved. This is however not the case starting from adding NLO corrections to the $n=1$ processes.

As for the tree level case, we can however provide correction terms similar to the LoopSim nNLO corrections. In the presence of tree level matrix elements for up to $N$ additional partons, and one-loop corrections for up to $M<N$ additional partons we then arrive at the merged cross section,

$$
\begin{aligned}
\mathrm{d} \sigma_{N, M, \rho}^{\text {merged }}= & \sum_{k=0}^{N-1} \operatorname{PS}_{\rho}^{-1}\left[\left(\mathrm{~d} \sigma_{\rho}^{(0)}\left(\phi_{k}, q_{k}\right)\right.\right. \\
& \left.\left.\quad-\int_{\rho}^{q_{k}} \mathrm{~d} q_{k+1} \frac{\mathrm{d} \sigma_{\rho}^{(0)}\left(\phi_{k+1}, q_{k+1}\right)}{\mathrm{d} q_{k+1}} \Delta_{k}\left(q_{k+1} \mid q_{k}\right)\right) \Delta_{k-1}\left(q_{k}|\cdots| q_{0}\right)\right] \\
& +\mathrm{d} \sigma_{\rho}^{(0)}\left(\phi_{N}, q_{N}\right) \Delta_{N-1}\left(q_{N}|\cdots| q_{0}\right) \\
& +\sum_{k=0}^{M-1} \operatorname{PS}_{\rho}^{-1}\left[\left(\mathrm{~d} \sigma_{\rho}^{(1, \text { incl })}\left(\phi_{k}, q_{k}\right)\right.\right. \\
& \left.\left.\quad-\int_{\rho}^{q_{k}} \mathrm{~d} q_{k+1} \frac{\mathrm{d} \sigma_{\rho}^{(1, \text { incl })}\left(\phi_{k+1}, q_{k+1}\right)}{\mathrm{d} q_{k+1}} \Delta_{k}\left(q_{k+1} \mid q_{k}\right)\right) \Delta_{k-1}\left(q_{k}|\cdots| q_{0}\right)\right] \\
& +\mathrm{PS}_{\rho}^{-1}\left[\mathrm{~d} \sigma_{\rho}^{(1, \text { incl })}\left(\phi_{M}, q_{M}\right) \Delta_{M-1}\left(q_{M}|\cdots| q_{0}\right)\right]
\end{aligned}
$$


which, upon parton shower action, will provide a merged sample with NLO accuracy for up to $M$-jet observables, LO accuracy for up to $N$-jet observables, including resummation at whatever accuracy is provided by the parton shower. Here, we have denoted the NLO corrections to inclusive cross sections as

$$
\mathrm{d} \sigma_{\rho}^{(1, \text { incl })}\left(\phi_{n}, q_{n}\right)=\mathrm{d} \sigma_{\rho}^{(1)}\left(\phi_{n}, q_{n}\right)+\int_{0}^{q_{n}} \mathrm{~d} q_{n+1} \frac{\mathrm{d} \sigma^{(0)}\left(\phi_{n+1}, q_{n+1}\right)}{\mathrm{d} q_{n+1}} \theta\left(q_{n}-\rho\right) .
$$

Note that the action of $\mathrm{PS}_{\rho}^{-1}$ integrates to one, which clarifies once more that we are able to preserve inclusive cross sections. Given the extensive discussions on reclustering, dynamic scale choices and the generation of the Sudakov weights in the context of merging approaches so far [7-13], we will not include a detailed algorithmic definition of the merging procedure here. Technical aspects will be subject to ongoing and future work concerned with the implementation of the procedure outlined here.

\section{Corrections below the merging scale}

Having derived the merged cross section in the presence of both tree level and one loop matrix elements, note that we actually have solved a NLO matching condition for each exclusive $n$-parton contribution above the merging scale, cf. eq. (5.2). More precisely,

- by preserving inclusive cross sections in the presence of only tree level matrix elements, we have matched each exclusive $n$-parton contribution to a LoopSim approximated $\mathrm{nLO}$, and

- by including exact NLO corrections, we have fulfilled this matching condition at NLO, while finally

- by preserving inclusive cross sections in the latter case, we start to generate approximate NNLO pieces, which could well be the basis for NNLO matching.

These considerations apply to contributions with emissions above the merging scale. Below the merging scale, we are still left with the shower approximation, and we will finally give a simple prescription of how nLO (in the case of tree level merging) and NLO (in the case of one loop merging) accuracy can be achieved also below the merging scale. To be precise, we consider the cases of $n$ partons above the merging scale and the first order contribution to zero or one emission below the merging scale. Demanding the required nLO or NLO accuracy, we obtain generalizations of the familiar NLO matching corrections restricted to the phase space for one emission below the merging scale,

$$
\begin{aligned}
\mathrm{d} \sigma_{N, \mu \mid \rho}^{\text {match }}= & \sum_{k=0}^{N-1} \operatorname{PS}_{\rho}^{-1}\left[\left(\mathrm{~d} \sigma_{\mu \mid \rho}^{(0), \text { match }}\left(\phi_{k}, q_{k}\right)\right.\right. \\
& \left.\left.\quad-\int_{\mu}^{\rho} \mathrm{d} q_{k+1} \frac{\mathrm{d} \sigma_{\mu \mid \rho}^{(0), \text { match }}\left(\phi_{k+1}, q_{k+1}\right)}{\mathrm{d} q_{k+1}} \Delta_{k}\left(q_{k+1} \mid q_{k}\right)\right) \Delta_{k-1}\left(q_{k}|\cdots| q_{0}\right)\right] \\
& +\mathrm{d} \sigma_{\mu \mid \rho}^{(0), \text { match }}\left(\phi_{N}, q_{N}\right) \Delta_{N-1}\left(q_{N}|\cdots| q_{0}\right)
\end{aligned}
$$


with

$$
\begin{aligned}
\mathrm{d} \sigma_{\mu \mid \rho}^{(0), \text { match }}\left(\phi_{n}, q_{n}\right)= & \mathrm{d} \sigma_{\mu}^{(0)}\left(\phi_{n}, q_{n}\right) \theta\left(q_{n-1}-\rho\right) \theta\left(\rho-q_{n}\right) \\
& -\mathrm{d} \sigma_{\rho}^{(0)}\left(\phi_{n-1}, q_{n-1}\right) \frac{\mathrm{d} \phi_{n}}{\mathrm{~d} \phi_{n-1}} P_{\mu \mid \rho}\left(\phi_{n-1}, q_{n}\right) .
\end{aligned}
$$

Note that these corrections do not change inclusive cross sections as is the case for plain NLO matching. Within these contributions, the parton shower cutoff can be sent to zero provided the singly unresolved limits are reproduced properly; a finite shower cutoff will act similar to a phase space slicing parameter in terms of which the NLO cross section is reproduced.

\section{Conclusions and outlook}

We have presented an extension to multileg matrix element and parton shower merging, which preserves inclusive cross sections at the level of the available accuracy, particularly at tree and one-loop level. This constraint is of utmost importance particularly for the latter case, as NLO corrections to shower splitting kernels are so far out of reach. This lack of shower accuracy manifests itself in terms of potentially large logarithmic contributions which are of the same order of magnitude as the NLO corrections tackled in recent approaches of combining fixed order corrections and parton shower resummation, thus spoiling NLO accuracy for lower jet multiplicities.

The formalism used to derive the modified algorithm is general enough to study the inclusion of even higher order corrections by successively replacing virtual contributions as approximated by the shower through their exact counterpart. The ingredients at for tree level and one loop merging, respectively, are given by fully differential LO calculations and inclusive NLO corrections differential in the respective Born variables. An implementation of the algorithm presented is subject to ongoing and future work.

Note added. During completion of this work a very similar approach to the problem by Lönnblad and Prestel came to the attention of the author, first results of which have been presented for the tree level case in [38] and for the one-loop case in [39].

\section{Acknowledgments}

This work was supported by the Helmholtz Alliance "Physics at the Terascale". The author wants to thank Johannes Bellm, Leif Lönnblad, Stefan Prestel and Frank Tackmann for fruitful discussion on the subject. The author acknowledges the kind hospitality of the Galileo Galilei Institute for Theoretical Physics where first studies connected to the present work have been carried out.

Open Access. This article is distributed under the terms of the Creative Commons Attribution License which permits any use, distribution and reproduction in any medium, provided the original author(s) and source are credited. 


\section{References}

[1] T. Sjöstrand, S. Mrenna and P.Z. Skands, A brief introduction to PYTHIA 8.1, Comput. Phys. Commun. 178 (2008) 852 [arXiv:0710.3820] [InSPIRE].

[2] M. Bähr et al., HERWIG++ physics and manual, Eur. Phys. J. C 58 (2008) 639 [arXiv:0803.0883] [INSPIRE].

[3] T. Gleisberg et al., SHERPA 1.alpha: a proof of concept version, JHEP 02 (2004) 056 [hep-ph/0311263] [INSPIRE].

[4] M.H. Seymour, Matrix element corrections to parton shower algorithms, Comput. Phys. Commun. 90 (1995) 95 [hep-ph/9410414] [INSPIRE].

[5] E. Norrbin and T. Sjöstrand, QCD radiation off heavy particles, Nucl. Phys. B 603 (2001) 297 [hep-ph/0010012] [InSPIRE].

[6] S. Catani, F. Krauss, R. Kuhn and B. Webber, QCD matrix elements + parton showers, JHEP 11 (2001) 063 [hep-ph/0109231] [INSPIRE].

[7] L. Lönnblad, Correcting the color dipole cascade model with fixed order matrix elements, JHEP 05 (2002) 046 [hep-ph/0112284] [INSPIRE].

[8] F. Krauss, Matrix elements and parton showers in hadronic interactions, JHEP 08 (2002) 015 [hep-ph/0205283] [INSPIRE].

[9] S. Hoeche et al., Matching parton showers and matrix elements, hep-ph/0602031 [INSPIRE].

[10] N. Lavesson and L. Lönnblad, Merging parton showers and matrix elements: Back to basics, JHEP 04 (2008) 085 [arXiv:0712.2966] [INSPIRE].

[11] S. Hoeche, F. Krauss, S. Schumann and F. Siegert, QCD matrix elements and truncated showers, JHEP 05 (2009) 053 [arXiv:0903.1219] [INSPIRE].

[12] K. Hamilton, P. Richardson and J. Tully, A modified CKKW matrix element merging approach to angular-ordered parton showers, JHEP 11 (2009) 038 [arXiv:0905.3072] [INSPIRE].

[13] L. Lönnblad and S. Prestel, Matching tree-level matrix elements with interleaved showers, JHEP 03 (2012) 019 [arXiv: 1109.4829] [INSPIRE].

[14] M. Dobbs, Phase space veto method for next-to-leading order event generators in hadronic collisions, Phys. Rev. D 65 (2002) 094011 [hep-ph/0111234] [InSPIRE].

[15] S. Frixione and B.R. Webber, Matching NLO QCD computations and parton shower simulations, JHEP 06 (2002) 029 [hep-ph/0204244] [INSPIRE].

[16] P. Nason, A New method for combining NLO QCD with shower Monte Carlo algorithms, JHEP 11 (2004) 040 [hep-ph/0409146] [INSPIRE].

[17] Z. Nagy and D.E. Soper, Matching parton showers to NLO computations, JHEP 10 (2005) 024 [hep-ph/0503053] [INSPIRE].

[18] S. Frixione, F. Stoeckli, P. Torrielli and B.R. Webber, NLO QCD corrections in HERWIG++ with MC@NLO, JHEP 01 (2011) 053 [arXiv:1010.0568] [INSPIRE].

[19] S. Platzer and S. Gieseke, Dipole showers and automated NLO matching in HERWIG++, Eur. Phys. J. C 72 (2012) 2187 [arXiv:1109.6256] [INSPIRE].

[20] Z. Nagy and D.E. Soper, A new parton shower algorithm: shower evolution, matching at leading and next-to-leading order level, hep-ph/0601021 [INSPIRE]. 
[21] J.-C. Winter and F. Krauss, Initial-state showering based on colour dipoles connected to incoming parton lines, JHEP 07 (2008) 040 [arXiv:0712.3913] [INSPIRE].

[22] M. Dinsdale, M. Ternick and S. Weinzierl, Parton showers from the dipole formalism, Phys. Rev. D 76 (2007) 094003 [arXiv:0709.1026] [InSPIRE].

[23] S. Schumann and F. Krauss, A parton shower algorithm based on Catani-Seymour dipole factorisation, JHEP 03 (2008) 038 [arXiv:0709.1027] [INSPIRE].

[24] W.T. Giele, D.A. Kosower and P.Z. Skands, A simple shower and matching algorithm, Phys. Rev. D 78 (2008) 014026 [arXiv:0707.3652] [INSPIRE].

[25] S. Platzer and S. Gieseke, Coherent parton showers with local recoils, JHEP 01 (2011) 024 [arXiv:0909.5593] [INSPIRE].

[26] W. Kilian, J. Reuter, S. Schmidt and D. Wiesler, An analytic initial-state parton shower, JHEP 04 (2012) 013 [arXiv:1112.1039] [INSPIRE].

[27] C.W. Bauer, F.J. Tackmann and J. Thaler, GenEvA. I. A new framework for event generation, JHEP 12 (2008) 010 [arXiv:0801.4026] [INSPIRE].

[28] C.W. Bauer, F.J. Tackmann and J. Thaler, GenEvA. II. A phase space generator from a reweighted parton shower, JHEP 12 (2008) 011 [arXiv:0801.4028] [INSPIRE].

[29] K. Hamilton and P. Nason, Improving NLO-parton shower matched simulations with higher order matrix elements, JHEP 06 (2010) 039 [arXiv: 1004.1764] [INSPIRE].

[30] S. Hoche, F. Krauss, M. Schonherr and F. Siegert, NLO matrix elements and truncated showers, JHEP 08 (2011) 123 [arXiv: 1009.1127] [INSPIRE].

[31] N. Lavesson and L. Lönnblad, Extending CKKW-merging to one-loop matrix elements, JHEP 12 (2008) 070 [arXiv:0811.2912] [INSPIRE].

[32] S. Hoeche, F. Krauss, M. Schonherr and F. Siegert, QCD matrix elements + parton showers: the NLO case, JHEP 04 (2013) 027 [arXiv: 1207.5030] [INSPIRE].

[33] T. Gehrmann, S. Hoche, F. Krauss, M. Schonherr and F. Siegert, NLO QCD matrix elements + parton showers in $e^{+} e^{-} \rightarrow$ hadrons, JHEP 01 (2013) 144 [arXiv:1207.5031] [INSPIRE].

[34] R. Frederix and S. Frixione, Merging meets matching in MC@NLO, JHEP 12 (2012) 061 [arXiv: 1209.6215] [INSPIRE].

[35] F. Tackmann, private communication.

[36] S. Plätzer, Parton showers and radiative corrections in QCD, Ph.D. Thesis, Karlsruhe Institute of Technology, Karlsruhe, Germany (2010).

[37] M. Rubin, G.P. Salam and S. Sapeta, Giant QCD K-factors beyond NLO, JHEP 09 (2010) 084 [arXiv:1006.2144] [INSPIRE].

[38] L. Lönnblad and S. Prestel, Unitarising matrix element + parton shower merging, JHEP 02 (2013) 094 [arXiv: 1211.4827] [INSPIRE].

[39] L. Lönnblad and S. Prestel, Merging multi-leg NLO matrix elements with parton showers, JHEP 03 (2013) 166 [arXiv:1211.7278] [INSPIRE]. 